\title{
SHADOWS OF CONVEX BODIES
}

\author{
KEITH BALL
}

\begin{abstract}
It is proved that if $C$ is a convex body in $\mathbb{R}^{n}$ then $C$ has an affine image $\widetilde{C}$ (of nonzero volume) so that if $P$ is any 1-codimensional orthogonal projection,$$
|P \widetilde{C}| \geq|\widetilde{C}|^{(n-1) / n} .
$$

It is also shown that there is a pathological body, $K$, all of whose orthogonal projections have volume about $\sqrt{n}$ times as large as $|K|^{(n-1) / n}$.
\end{abstract}

\section{INTRODUCTION}

The problems discussed in this paper concern the areas of shadows (orthogonal projections) of convex bodies and, to a lesser extent, the surface areas of such bodies. If $C$ is a convex body in $\mathbb{R}^{n}$ and $\theta$ a unit vector, $P_{\theta} C$ will denote the orthogonal projection of $C$ onto the 1-codimensional space perpendicular to $\theta$. Volumes and areas of convex bodies and their surfaces will be denoted with $|\cdot|$.

The relationship between shadows and surface areas of convex bodies is expressed in Cauchy's well-known formula. For each $n \in \mathbf{N}$, let $v_{n}$ be the volume of the $n$-dimensional Euclidean unit ball and let $\sigma=\sigma_{n-1}$ be the rotationally invariant probability on the unit sphere $S^{n-1}$. Cauchy's formula states that if $C$ is a convex body in $\mathbb{R}^{n}$ then its surface area is

$$
|\partial C|=\frac{n v_{n}}{v_{n-1}} \int_{S^{n-1}}\left|P_{\theta} C\right| d \sigma(\theta) .
$$

The classical isoperimetric inequality in $\mathbb{R}^{n}$ states that any body has surface area at least as large as a Euclidean ball of the same volume. The first section of this paper is devoted to the proof of a "local" isoperimetric inequality showing that all bodies have large shadows rather than merely large surface area (or average shadow). The principal motivation for this result is its relationship to a conjecture of Vaaler and the important problems surrounding it. This theorem and its connection with Vaaler's conjecture are described at the beginning of $\S 1$.

An important role is played in the theory of convex bodies by the so-called "projection body" of a convex body. It is easily seen, by considering polytopes,

Received by the editors September 12, 1989.

1980 Mathematics Subject Classification (1985 Revision). Primary 52A20, 52A40.

The author was supported in part by NSF DMS- 8807243 . 
that for every convex body $C \subset \mathbb{R}^{n}$, there is a Borel measure $\mu$ on $S^{n-1}$ so that for each $\theta \in S^{n-1}$,

$$
\left|P_{\theta} C\right|=\int_{S^{n-1}}|\langle\theta, \phi\rangle| d \mu(\phi)
$$

Hence, there is a norm $\|\cdot\|$ on $\mathbb{R}^{n}$, with $\|\theta\|=\left|P_{\theta} C\right|$ for each $\theta \in S^{n-1}$, with respect to which $\mathbb{R}^{n}$ is isometrically isomorphic to a subspace of $L_{1}$. The unit ball of this norm is a symmetric convex body which will be denoted $\Pi^{*}(C)$. The map $\Pi^{*}$, from the collection of convex bodies in $\mathbb{R}^{n}$ to the collection of unit balls of representations of $n$-dimensional subspaces of $L_{1}$ on $\mathbb{R}^{n}$, has been extensively studied: see e.g. [B-L]. The restriction of $\Pi^{*}$ to the class of centrally symmetric convex bodies was shown to be injective by Aleksandrov: if $C$ and $D$ are centrally symmetric convex bodies and $\left|P_{\theta} C\right|=\left|P_{\theta} D\right|$ for all $\theta \in S^{n-1}$ then $C=D$. If the condition of central symmetry is dropped, $C$ and $D$ may not even be congruent: The Rouleaux triangle in $\mathbb{R}^{2}$ has all 1-dimensional shadows equal in length to those of some disc.

The map $\Pi^{*}$ was shown to be surjective by Minkowski. What Minkowski's proof actually gives (at least in the context of polytopes) is the result stated in $\S 2$ as Lemma 6. (This rather detailed statement of Minkowski's theorem will be needed for the construction of a pathological body with large shadows.)

An important observation of Petty [P], is that if $T$ is a linear operator on $\mathbb{R}^{n}$ of determinant 1 then, for every $C$,

$$
\Pi^{*}(T C)=T\left(\Pi^{*}(C)\right) .
$$

Motivated in part by Aleksandrov's theorem on the injectivity of $\Pi^{*}$, Shephard asked whether, if $C$ and $D$ are centrally symmetric convex bodies with

$$
\left|P_{\theta} C\right| \geq\left|P_{\theta} D\right| \text { for all } \theta \in S^{n-1}
$$

then necessarily $|C| \geq|D|$. This question was answered in the negative by Petty and Schneider independently in [P and S]. (The corresponding question for sections rather than shadows was posed in [B-P] and answered (again in the negative) by [L-R].) The second section of this paper contains a strongly negative answer to Shephard's question. It will be shown that a "random" $n$ dimensional subspace of $l_{\infty}^{2 n}$ has a unit ball, all of whose shadows are very large compared with those of a Euclidean ball of the same volume. Such examples suggest that the Shephard problem is less delicate than the Busemann-Petty problem for sections: it is an important open question as to whether there are highly pathological examples for the latter problem. This question is usually referred to as the slicing problem.

\section{A LOCAL ISOPERIMETRIC INEQUALITY}

In [V], Vaaler conjectured that for every $n \in \mathbf{N}$, every symmetric convex body $C \subset \mathbb{R}^{n}$ and every $k<n$, there is an affine image $T C$ of $C$ (for some 
automorphism $T$ of $\mathbb{R}^{n}$ ) so that for every $k$-dimensional subspace $H$ of $\mathbb{R}^{n}$,

$$
|H \cap T C| \geq|T C|^{k / n} \text {. }
$$

(Vaaler actually conjectured something slightly stronger, which is false for small values of $k$.) This conjecture strengthens the slicing problem mentioned above, namely: there exists a $\delta>0$ so that for each $n$ and $C$ there is a 1-codimensional subspace $H$ of $\mathbb{R}^{n}$ with

$$
|H \cap C| \geq \delta|C|^{(n-1 / n} .
$$

The case $k=1$ of Vaaler's conjecture (for arbitrary $n$ and $C$ ) was proved by the present author in $\left[B_{1}\right]$, where the result is stated as a volume ratio estimate. The proof for $k=1$ really estimates volumes of 1-dimensional shadows and then uses the fact that the smallest 1-dimensional section of a convex body is its smallest 1-dimensional shadow. Since minimal sections and minimal shadows are not identical for subspaces of dimension larger than 1, such an argument cannot be employed if $k>1:$ but it is natural to ask whether Vaaler's conjecture can be proved for shadows (of dimension other than 1) independently of the outstanding problem for sections. The principal result of this paper deals with the most important case,

$$
k=n-1 .
$$

Theorem 1. Let $C$ be a convex body in $\mathbb{R}^{n}$. There is an affine image $\widetilde{C}$ of $C$ (with nonzero volume) so that for each unit vector $\theta \in \mathbb{R}^{n}$,

$$
\left|P_{\theta} \widetilde{C}\right| \geq|\widetilde{C}|^{(n-1) / n} \text {. }
$$

The result is exactly best possible as shown by the cube.

The proof of Theorem 1 uses the well-known theorem of John [J] which characterises ellipsoids of minimal volume containing convex bodies. This result is stated here as a lemma.

Lemma 2. Let $K$ be a symmetric convex body in $\mathbb{R}^{n}$. The ellipsoid of minimal volume containing $K$ is the Euclidean unit ball $B_{2}^{n}$ if and only if $K$ is contained in $B_{2}^{n}$ and there are Euclidean unit vectors $\left(u_{i}\right)_{1}^{m}$ (for some $m \in \mathbf{N}$ ) on the boundary $\partial K$ of $K$ and positive numbers $\left(c_{i}\right)_{1}^{m}$ so that

$$
\sum_{1}^{m} c_{i} u_{i} \otimes u_{i}=I_{n}
$$

(Here, $u_{i} \otimes u_{i}$ is the usual rank-M1 orthogonal projection onto the span of $u_{i}$ and $I_{n}$ is the identity on $\mathbb{R}^{n}$.) The identity above states that the $u_{i}$ 's are distributed rather like an orthonormal basis in that for each $x \in \mathbb{R}^{n}$,

$$
|x|^{2}=\sum_{1}^{m} c_{i}\left\langle u_{i}, x\right\rangle^{2}
$$

The equality of the traces of the operators appearing above shows that $\sum_{1}^{m} c_{i}=n$. 
Theorem 1 will be deduced from the following, which is little more than an affine invariant reformulation.

Theorem 3. Suppose $C$ is a convex body in $\mathbb{R}^{n},\left(u_{i}\right)_{1}^{m}$ a sequence of unit vectors in $\mathbb{R}^{n}$ and $\left(c_{i}\right)_{1}^{m}$ a sequence of positive numbers for which

$$
\sum_{1}^{m} c_{i} u_{i} \otimes u_{i}=I_{n}
$$

For each $i$, let $P_{i}$ be the orthogonal projection $P_{u_{i}}$ along $u_{i}$. Then

$$
|C|^{n-1} \leq \prod_{1}^{m}\left|P_{i} C\right|^{c_{i}}
$$

There is an obvious relationship between Theorem 3 and Lemma 2. Theorem 3 is closely related to an inequality of Brascamp and Lieb $[\mathrm{Br}-\mathrm{L}]$ which has been used in several places by this author, $\left[\mathrm{B}_{1}\right.$ and $\left.\mathrm{B}_{2}\right]$. Theorem 3 and generalisations of it were conjectured in $[\mathrm{Br}-\mathrm{L}]$ (in a different form). The special case of Theorem 3 in which the $u_{i}$ 's form an orthonormal basis (in which case, necessarily, $c_{i}=1$ for $1 \leq i \leq m=n$ ) was proved by Loomis and Whitney [L-W]. Theorem 3 can be regarded as an isoperimetric inequality in that it estimates the volume of a body in terms of an average of volumes of its shadows: in this case, a geometric average. The key point is that the "number" of shadows involved is small enough that the local isoperimetric inequality of Theorem 1 can be deduced.

Proof of Theorem 1. Because of the intertwining property of $\Pi^{*}$ with linear transformations (1), there is an affine image $\widetilde{C}$ of $C$ so that the ellipsoid of minimal volume containing $\Pi^{*}(\widetilde{C})$ is the Euclidean ball $B_{2}^{n}$. This ensures that

$$
\left|P_{\theta}(\widetilde{C})\right| \geq 1
$$

for every unit vector $\theta \in \mathbb{R}^{n}$ and, by Lemma 2 , that there are unit vectors $\left(u_{i}\right)_{1}^{m}$ and positive numbers $\left(c_{i}\right)_{1}^{m}$ so that

$$
\left|P_{u_{i}} \widetilde{C}\right|=1 \text { for each } i
$$

and $\sum_{1}^{m} c_{i} u_{i} \otimes u_{i}=I_{n}$.

Now, from Theorem 3,

$$
|\widetilde{C}|^{n-1} \leq \prod_{1}^{m}\left|P_{u_{i}} \widetilde{C}\right|^{c_{i}}=1 .
$$

The proof of Theorem 3 uses Minkowski's inequality for mixed volumes to establish a duality between the 1-codimensional problem to be solved and a 1dimensional problem. The relevant information on mixed volumes is included here for completeness. 
For a fixed $n$, let $\mathscr{C}=\mathscr{C}_{n}$ be the set of compact, convex subsets of $\mathbb{R}^{n} . \mathscr{C}$ can be regarded as a convex cone under Minkowski addition and multiplication by nonnegative scalars. A crucial theorem of Minkowski states that there is a symmetric $n$-positive-linear form

$$
V: \underbrace{\mathscr{C} \times \cdots \times \mathscr{C}}_{n \text { times }} \rightarrow[0, \infty)
$$

whose diagonal is volume: i.e. $V$ is positive linear in each of its $n$ arguments and, for each $C \in \mathscr{C}$,

$$
|C|=V(C, \ldots, C) .
$$

The values of $V$ are called mixed volumes. As a consequence of Minkowski's theorem, the volume $|C+t D|,(t \in[0, \infty))$ can be expanded as a polynomial in $t$,

$$
|C+t D|=\sum_{0}^{n}\left(\begin{array}{l}
n \\
k
\end{array}\right) v_{n-k}(C, D) t^{k}
$$

where

$$
v_{n-k}(C, D)=V \underbrace{C, \cdots, C}_{n-k}, \underbrace{D, \ldots, D}_{k})
$$

and is called the $n-k$ th mixed volume of $C$ and $D$.

The Brunn-Minkowski inequality states that

$$
|C+t D|^{1 / n}
$$

is a concave function of $t($ on $[0, \infty))$. Differentiation of $(2)$ at $t=0$ gives Minkowski's inequality

$$
|C|^{(n-1) / n}|D|^{1 / n} \leq v_{n-1}(C, D) .
$$

If $D=B_{2}^{n}$ is the Euclidean unit ball, (3) is the classical isoperimetric inequality. Inequality (3) will be used here with an appropriate choice of $D$.

A Minkowski sum of line segments

$$
\begin{array}{r}
\sum_{1}^{m}\left[-x_{i}, x_{i}\right]=\left\{x \in \mathbb{R}^{n}: x=\sum_{1}^{m} \lambda_{i} x_{i} \text { for some sequence }\left(\lambda_{i}\right)_{1}^{m}\right. \\
\text { with } \left.\left|\lambda_{i}\right| \leq 1,1 \leq i \leq m\right\}
\end{array}
$$

is called a zonotope. It is easily checked that if $u$ is a unit vector and $D=$ $[-u, u]=\left\{x \in \mathbb{R}^{n}: x=\lambda u\right.$ for some $\left.\lambda \in[-1,1]\right\}$ then

$$
v_{n-1}(C, D)=\frac{2}{n}\left|P_{u} C\right|
$$

for any convex body $C$. So if $Z$ is the zonotope

$$
Z=\sum_{1}^{m} \alpha_{i}\left[-u_{i}, u_{i}\right]
$$


with $\left(u_{i}\right)_{1}^{m}$ a sequence of unit vectors and $\left(\alpha_{i}\right)_{1}^{m}$ a sequence of positive numbers,

$$
v_{n-1}(C, Z)=\frac{2}{n} \sum_{1}^{m} \alpha_{i}\left|P_{u_{i}} C\right|
$$

There is equality in Minkowski's inequality (3) if $C=D$ and so with $Z$ as before

$$
|Z|=\frac{2}{n} \sum_{1}^{m} \alpha_{i}\left|P_{u_{i}} Z\right|
$$

This identity is usually called the volume formula for zonotopes. A simple induction argument can be used to obtain an expression for $|Z|$ in terms of the $\alpha_{i}$ 's and determinants of square matrices formed from the $u_{i}$ 's. In the following lemma, a similar inductive argument is used to obtain an estimate for $|Z|$ which is easier to use than the actual value.

Lemma 4. Let $\left(u_{i}\right)_{1}^{m}$ be a sequence of unit vectors in $\mathbb{R}^{n},\left(c_{i}\right)_{1}^{m}$ a sequence of positive numbers with

$$
\sum_{1}^{m} c_{i} u_{i} \otimes u_{i}=I_{n}
$$

and $\left(\alpha_{i}\right)_{1}^{m}$ another sequence of positive numbers. If

$$
Z=\sum_{1}^{m} \alpha_{i}\left[-u_{i}, u_{i}\right]
$$

then

$$
|Z| \geq 2^{n} \prod_{1}^{m}\left(\frac{\alpha_{i}}{c_{i}}\right)^{c_{i}}
$$

Proof. The proof uses induction on the dimension $n$. For $n=1$,

$$
|Z|=2 \sum_{1}^{m} \alpha_{i}=2 \sum_{1}^{m} c_{i}\left(\frac{\alpha_{i}}{c_{i}}\right) \geq 2 \prod_{1}^{m}\left(\frac{\alpha_{i}}{c_{i}}\right)^{c_{i}}
$$

by the AM-GM inequality since $\sum_{1}^{m} c_{i}=1$, when $n=1$.

For larger $n$, the volume formula (5) shows that with $P_{i}=P_{u_{i}}$

$$
\begin{aligned}
|Z| & =\frac{2}{n} \sum_{1}^{m} \alpha_{i}\left|P_{i} Z\right|=2 \sum_{1}^{m} \frac{c_{i}}{n} \frac{\alpha_{i}}{c_{i}}\left|P_{i} Z\right| \\
& \geq 2 \prod_{1}^{m}\left(\frac{\alpha_{i}}{c_{i}}\left|P_{i} Z\right|\right)^{c_{i} / n}
\end{aligned}
$$

since $\sum_{1}^{m} c_{i}=n$.

Now, for each fixed $i, P_{i} Z$ is a zonotope with summands

$$
\alpha_{j}\left[-P_{i}\left(u_{j}\right), P_{i}\left(u_{j}\right)\right], \quad 1 \leq j \leq m,
$$


and contained in the $(n-1)$-dimensional space $P_{i}\left(\mathbb{R}^{n}\right)$. For each $i$ and $j$ let

$$
\gamma_{i j}=\left|P_{i}\left(u_{j}\right)\right|=\left|P_{j}\left(u_{i}\right)\right|
$$

(so $\gamma_{i j}^{2}=1-\left\langle u_{i}, u_{j}\right\rangle^{2}$ ). Then for each $i$,

$$
P_{i} Z=\sum_{j=1}^{m} \alpha_{j} \gamma_{i j}\left[-v_{i j}, v_{i j}\right]
$$

where $v_{i j}$ is the unit vector in the direction of $P_{i} u_{j}$ (or any direction if $\gamma_{i j}=0$ ). Now, for each $i$,

$$
P_{i}=\sum_{j=1}^{m} c_{j} P_{i} u_{j} \otimes P_{i} u_{j}=\sum_{j=1}^{m} c_{j} \gamma_{i j}^{2} v_{i j} \otimes v_{i j}
$$

and $P_{i}$ acts as the identity on $P_{i}\left(\mathbb{R}^{n}\right)$. So, by the inductive hypothesis,

$$
\left|P_{i} Z\right| \geq 2^{n-1} \prod_{j=1}^{m}\left(\frac{\alpha_{j} \gamma_{i j}}{c_{j} \gamma_{i j}^{2}}\right)^{c_{j} \gamma_{i j}^{2}}
$$

where it is understood that if $\gamma_{i j}=0$, the $j$ th factor is 1: (so in particular, the $i$ th factor is 1 ).

Substitution of the inequalities for each $i$ into (6) shows that

$$
|Z| \geq 2^{n}\left(\prod_{i, j=1}^{m}\left(\frac{\alpha_{i}}{c_{i}}\right)^{c_{i}}\left(\frac{\alpha_{j}}{c_{j} \gamma_{i j}}\right)^{c_{i} c_{j} \gamma_{i j}^{2}}\right)^{1 / n}
$$

and this expression is at least

$$
2^{n} \prod_{i=1}^{m}\left(\frac{\alpha_{i}}{c_{i}}\right)^{c_{i}}
$$

because $1 / \gamma_{i j} \geq 1$ for all $i$ and $j$ and, for each $j$,

$$
\sum_{i=1}^{m} c_{i} \gamma_{i j}^{2}=\sum_{i=1}^{m} c_{i}\left(1-\left\langle u_{i}, u_{i}\right\rangle^{2}\right)=n-1 .
$$

Proof of Theorem 3. Let $C,\left(u_{i}\right)_{1}^{m},\left(c_{i}\right)_{1}^{m}$ and $\left(P_{i}\right)_{1}^{m}$ be as in the theorem's statement. For each $i$, set

$$
\alpha_{i}=\frac{c_{i}}{\left|P_{i} C\right|} .
$$

With $Z=\sum_{1}^{m} \alpha_{i}\left[-u_{i}, u_{i}\right],(3)$ and (4) above, show that

$$
|C|^{n-1} \leq|Z|^{-1}\left(\frac{2}{n} \sum_{1}^{m} \alpha_{i}\left|P_{i} C\right|\right)^{n}=|Z|^{-1}\left(\frac{2}{n} \sum_{1}^{m} c_{i}\right)^{n}=2^{n}|Z|^{-1}
$$

since $\sum_{1}^{m} c_{i}=n$. 
Now by Lemma 4,

$$
|C|^{n-1} \leq 2^{n}\left(2^{n} \prod_{i=1}^{m}\left(\frac{\alpha_{i}}{c_{i}}\right)^{c_{i}}\right)^{-1}=\prod_{i=1}^{m}\left|P_{i} C\right|^{c_{i}} .
$$

Remark 1. The lower estimate for volumes of zonotopes given by Lemma 4 could have been obtained from the volume ratio estimates proved in the author's paper $\left[\mathrm{B}_{2}\right]$ together with Reisner's reverse Santalo inequality for zonoids, $[R]$.

Remark 2. For every convex body $C$ in $\mathbb{R}^{n}$, there is an affine image $\tilde{C}$ of $C$ with

$$
\left|P_{\theta} \widetilde{C}\right| \leq M \sqrt{n}|\widetilde{C}|^{(n-1) / n}
$$

for each unit vector $\theta$ ( $M$ being an absolute constant). This estimate depends upon the fact that subspaces of $L_{1}$ have uniformly bounded volume ratios. It seems likely that $M$ could be taken to be 1 for symmetric convex bodies $C$ (the cube again being extremal). The estimates in $\left[\mathrm{B}_{2}\right]$ show that $M$ can be taken to be $2 \sqrt{e} / \pi \approx 1.05$ in this case.

\section{A Remark on the Shephard PRoblem}

This section contains a strongly negative solution to the Shephard problem described in the introduction. Petty and Schneider [P and S] constructed pairs of bodies $C$ and $D$ in $\mathbb{R}^{n}$ so that

$$
\left|P_{\theta} D\right| \leq\left|P_{\theta} C\right| \text { for all } \theta \in S^{n-1},
$$

but $|D|>|C|$. They also showed that the conclusion $|D| \leq|C|$ does hold if $C$ is a zonoid (a limit, in the Hausdorff metric, of zonotopes). A little more generally, if $C$ and $D$ are convex bodies satisfying (7) and $Z=\sum_{1}^{m} \alpha_{i}\left[-u_{i}, u_{i}\right]$ is a zonotope included in $C$ (with $u_{i} \in S^{n-1}, 1 \leq i \leq m$ ), then, rather as in the proof of Theorem 3 ,

$$
\begin{aligned}
|D|^{(n-1) / n}|Z|^{1 / n} & \leq v_{n-1}(D, Z)=\sum_{1}^{m} \alpha_{i}\left|P_{u_{i}} D\right| \\
& \leq \sum_{1}^{m} \alpha_{i}\left|P_{u_{i}} C\right|=v_{n-1}(C, Z) \\
& \leq v_{n-1}(C, C)=|C|
\end{aligned}
$$

where the last inequality is a consequence of the monotonicity of mixed volumes: (this particular case is obvious from the fact that if $Z \subset C$,

$$
C+t Z \subset(1+t) C
$$

for all $t \geq 0$ ).

Hence,

$$
|D| \leq\left(\frac{|C|}{|Z|}\right)^{1 /(n-1)}|C| .
$$


Since every convex body $C$ contains a zonoid $Z$ with $|C| /|Z|$ not too large, the last inequality shows that under hypothesis (7), one does have

$$
|D| \leq \frac{3}{2} \sqrt{n}|C| .
$$

Apart from a constant factor, it turns out that this is the most that can be said. The Euclidean ball of volume 1 in $\mathbb{R}^{n}$ has shadows of 1-codimensional volume about $\sqrt{e}($ as $n \rightarrow \infty)$ : in Theorem 5 it is shown that there is a body of volume 1 in $\mathbb{R}^{n}$, all of whose shadows have volume about $\sqrt{n}$.

Theorem 5. There is a constant $\delta>0$ so that for each $n \in \mathbf{N}$, there is a symmetric convex body $K$ in $\mathbb{R}^{n}$ satisfying,

$$
\left|P_{\theta} K\right| \geq \delta \sqrt{n}|K|^{(n-1) / n}
$$

for every unit vector $\theta \in \mathbb{R}^{n}$.

The proof of Theorem 5 depends heavily upon the theorem of Minkowski on the existence of bodies with given projections. An appropriately detailed statement of this theorem is given as Lemma 6 below: the following notation is needed. For a sequence $\left(u_{i}\right)_{1}^{m}$ of unit vectors spanning $\mathbb{R}^{n}$ and a sequence $\left(\gamma_{i}\right)_{1}^{m}$ of positive numbers let $\mathscr{F}=\mathscr{F}\left(\left(u_{i}\right),\left(\gamma_{i}\right)\right)$ be the family of convex bodies of the form

$$
\left\{x \in \mathbb{R}^{n}:\left|\left\langle x, u_{i}\right\rangle\right| \leq t_{i}, \quad 1 \leq i \leq m\right\}
$$

indexed by sequences $\left(t_{i}\right)_{1}^{m}$ of positive reals satisfying

$$
\sum_{1}^{m} \gamma_{i} t_{i}=1
$$

Lemma 6. With the above notation, $\mathscr{F}$ has a unique element of maximal volume, $K$ (say), satisfying

$$
\left|P_{\theta} K\right|=\frac{n|K|}{2} \sum_{1}^{m} \gamma_{i}\left|\left\langle u_{i}, \theta\right\rangle\right|
$$

for each $\theta \in S^{n-1}$.

The body that satisfies the conclusion of Theorem 5 will be the unit ball of a "random" $n$-dimensional subspace of $l_{\infty}^{2 n}$. Such subspaces are known to have many pathological properties stemming from the fact that the $l_{1}^{2 n}$ and $l_{2}^{2 n}$ norms are well-equivalent on such spaces: this was proved in [F-L-M]. For the history of this result and its many extensions, see e.g. [M-S]. The form of the result needed here is given as a lemma.

Lemma 7. There is a $\delta>0$ so that for $n \in \mathbf{N}$ there are unit vectors $u_{1}, \ldots, u_{2 n}$ in $\mathbb{R}^{n}$ with

$$
\sum_{1}^{2 n}\left|\left\langle x, u_{i}\right\rangle\right| \geq \delta \sqrt{n}|x|
$$

for every vector $x \in \mathbb{R}^{n}$. 
The last lemma that will be needed is a result of Vaaler [V], concerning volumes of sections of the cube in $\mathbb{R}^{n}$. The form required here is the following. Lemma 8. Let $\left(u_{i}\right)_{1}^{m}$ be a sequence of unit vectors in $\mathbb{R}^{n}$. Then the volume of the symmetric convex body with these vectors as boundary functionals satisfies

$$
\left|\left\{x \in \mathbb{R}^{n}:\left|\left\langle x, u_{i}\right\rangle\right| \leq 1, \quad 1 \leq i \leq m\right\}\right|^{1 / n} \geq 2 \sqrt{\frac{n}{m}} .
$$

Proof of Theorem 5. Let $\left(u_{i}\right)_{1}^{2 m}$ be a sequence of vectors with the property described in Lemma 7. Take $m=2 n$ and apply Lemma 6 with $\gamma_{i}=\frac{1}{m}$, $1 \leq i \leq m$.

The body $K$ of maximal volume in the family $\mathscr{F}$ satisfies

$$
\left|P_{\theta} K\right|=\frac{n|K|}{2 m} \sum_{1}^{m}\left|\left\langle u_{i}, \theta\right\rangle\right| \geq \frac{|K|}{4} \cdot \delta \sqrt{n}
$$

for every unit vector $\theta$. Now, $\mathscr{F}$ also contains the body

$$
C=\left\{x \in \mathbb{R}^{n}:\left|\left\langle x, u_{i}\right\rangle\right| \leq 1,1 \leq i \leq m\right\} .
$$

So (by the maximality of $K$ ), $|K| \geq|C|$. But, by Lemma 8 ,

$$
|C|^{1 / n} \geq 2 \sqrt{\frac{n}{m}}=\sqrt{2}
$$

and hence $|K|^{1 / n} \geq \sqrt{2}$. Therefore, for every unit vector $\theta$,

$$
\left|P_{\theta} K\right| \geq \frac{\delta \sqrt{n}}{2 \sqrt{2}}|K|^{(n-1) / n} \text {. }
$$

Remark. The above argument can be extended slightly to estimate surface area to volume ratios of subspaces of $l_{\infty}$. If $i q(X)$ is the isoperimetric quotient of the finite-dimensional normed space $X$ (see [Schü] for definitions) normalised so that $i q\left(l_{2}^{n}\right)=1$ then for every $n$-dimensional subspace $X$ of $l_{\infty}^{m}$,

$$
i q(X) \geq \frac{\delta \sqrt{n}}{\sqrt{1+\log \frac{m}{n}}}
$$

for some absolute constant $\delta>0$.

\section{REFERENCES}

[B $\left.\mathrm{B}_{1}\right] \quad$ K. M. Ball, Volumes of sections of cubes and related problems, Israel Seminar (G.A.F.A.) 1988, Lecture Notes in Math., vol. 1376, Springer-Verlag, Berlin and New York, 1989, pp. 251-260.

$\left[\mathrm{B}_{2}\right] \_$, Volumes ratios and a reverse isoperimetric inequality, In preparation.

[B-L] J. Bourgain and J. Lindenstrauss, Projection bodies, Israel Seminar (G.A.F.A) 1986-87, Lecture Notes in Math., vol. 1317, Springer-Verlag, Berlin and New York, 1988, pp. 250269.

[Br-L] Herm Jan Brascamp and Elliott H. Lieb, Best constants in Young's inequality, its converse and its generalization to more than three functions, Adv. in Math. 20 (1976), 151-173.

[B-P] H. Busemann and C. M. Petty, Problems on convex bodies, Math. Scand. 4 (1956), 88-94. 
[F-L-M] T. Figiel, J. Lindenstrauss and V. D. Milman, The dimension of almost spherical sections of convex bodies, Acta Math. 139 (1977), 53-94.

[J] F. John, Extremum problems with inequalities as subsidiary conditions, Courant Anniversary Volume, Interscience, New York, 1948, pp. 187-204.

[L-R] D. G. Larman and C. A. Rogers, The existence of a centrally symmetric convex body with central sections that are unexpectedly small, Mathematika 22 (1976), 164-175.

[L-W] L. H. Loomis and H. Whitney, An inequality related to the isoperimetric inequality, Bull. Amer. Math. Soc. 55 (1949), 961-962.

[M-S] V. D. Milman and G. Schechtman, Asymptotic theory of finite dimensional normed spaces, Lecture Notes in Math., vol. 1200, Springer-Verlag, Berlin and New York, 1986, pp. 64-104.

[P] C. M. Petty, Projection bodies, Proc. Colloq. on Convexity, Copenhagen, 1967, pp. 234-241.

[R] S. Reisner, Zonoids with minimal volume product, Math. Z. 192 (1986), 339-346.

[S] R. Schneider, Zu einem Problem von Shephard über die Projektionen konvexer Körper, Math. Z. 101 (1967), 71-82.

[Schü] C. Schütt, The isoperimetric quotient and some classical Banach spaces, (to appear).

[V] J. D. Vaaler, A geometric inequality with applications to linear forms, Pacific J. Math. 83 (1979), 543-553.

Department of Mathematics, Trinity College, Cambridge, England

Department of Mathematics, Texas A\&M University, College Station, Texas 77843

Current address: Department of Mathematics, U.C.L., Gower Street, London WC1E, England 